\title{
Correction to: Facial swelling for the emergency radiologist-typical and atypical causes
}

\author{
Stephen M. Smith ${ }^{1}$ - Jarett C. Thelen ${ }^{1}$ - Alok A. Bhatt ${ }^{2}$ - Alexander T. Kessler ${ }^{1}$ \\ Published online: 30 June 2020 \\ (C) American Society of Emergency Radiology 2020
}

\section{Correction to: Emergency Radiology (2020) https://doi.org/10.1007/s10140-020-01809-x}

The above article was published online with inverted figures legends for Fig. 1-6. The correct presentation is shown below. The original article has been corrected.

Fig. 1 legend: Myositis. Axial CECT image in a 65-year-old female after right maxillary root canal demonstrates asymmetric enlargement/hypoenhancement of the right temporalis and pterygoid musculature (arrows) with surrounding inflammatory stranding.

Fig. 2 legend: Buccal space abscess. Sagittal (a) demonstrates periapical hypoattenuation within a left maxillary molar (arrowhead). Axial NECT (b) demonstrates a large multiloculated fluid collection extending into the buccal space (arrow). Axial T1 fat-suppressed post-contrast image demonstrates thick rimenhancement of the collection compatible with abscess.

Fig. 3 legend: Ludwig's angina. Axial (a) and coronal (b) CECT images demonstrate ill-defined fluid and locules of air (arrows) within the right mouth floor. Sagittal (c) image in the bone window demonstrates odontogenic infection of a right mandibular molar (arrowhead), presumably the origin of

The online version of the original article can be found at https://doi.org/ 10.1007/s10140-020-01809-x

Alexander T. Kessler

Alex_Kessler@urmc.rochester.edu

1 Department of Imaging Sciences, University of Rochester Medical, Center, 601 Elmwood Avenue, P.O. Box 648, Rochester, NY 14642, USA

2 Department of Radiology, Mayo Clinic, 4500 San Pablo Road S, Jacksonville, FL 32224, USA infection. An endotracheal tube is present due to compromised airway patency.

Fig. 4 legend: Acute sialadenitis. Axial contrast-enhanced CT image demonstrates asymmetric enlargement and enhancement of the left parotid gland (arrow).

Fig. 5 legend: Preseptal Orbital Cellulitis. Axial (a) and sagittal (b) contrastenhanced CT (CECT) images demonstrate asymmetric edema and stranding within the preseptal soft tissues of the left orbit (arrows). The intraorbital fat is preserved. Fig. 6 legend: Postseptal orbital cellulitis. Axial CECT (a), axial T2 fat suppressed (b), and axial T1 fat suppressed post-contrast (c) images demonstrate edema and stranding within the preseptal soft tissues (arrowheads), as well as the intraconal fat (long arrows). There is also asymmetric edema/ enhancement within the subdural space along the right middle cranial fossa (short arrows) compatible with intracranial spread of infection.

Publisher's note Springer Nature remains neutral with regard to jurisdictional claims in published maps and institutional affiliations. 